\title{
Las funciones ejecutivas y la lectura: Revisión sistemática de la literatura ${ }^{6}$
}

\author{
Gerardo Restrepo \\ Médico Neurólogo Infantil \\ Université de Sherbrooke \\ Quebec, Canadá \\ Correo electrónico: Gerardo.Restrepo@USherbrooke.ca
}

Liliana Calvachi Gálvez

Magister en Gestión y desarrollo de los Recursos Humanos

Universidad de Nariño

Pasto, Colombia

Correo electrónico: clinicas.udenar@gmail.com
Isabel Cristina Cano Álvarez
Magíster en Psicología Institución Universitaria CESMAG
Pasto, Colombia

Correo electrónico: iccano@iucesmag.edu.co

\section{Adalberto León Ruiz Márquez}

Phd (c) En educación y Psicología Institución Universitaria CESMAG

Pasto, Colombia

Correo electrónico: alruiz@iucesmag.edu.co
Recibido: $16 / 08 / 2018$

Evaluado: 08/10/2018

Aceptado: 09/11/2018

\section{Resumen}

Las funciones ejecutivas son procesos cognitivos de alto nivel, responsables de la planeación, ejecución y evaluación que el sujeto hace de su propia conducta. Su estudio se justifica puesto que están involucradas en los procesos de aprendizaje y desarrollo infantil y son el núcleo mismo del comportamiento social y adaptativo del ser humano. Por consiguiente, identificar los factores que intervienen en su desarrollo se ha convertido en un objetivo esencial de las investigaciones que buscan desarrollar intervenciones eficaces, en el campo clínico y educativo, en niños y adolescentes. Este artículo revisa la literatura científica que relaciona las funciones ejecutivas y el desempeño académico con el aprendizaje de la lectura. Se revisan los artículos científicos sobre este tópico, publicados desde el 2010 hasta el 2015. De un total de 34 resultados de búsqueda, 6 publicaciones científicas fueron seleccionadas y analizadas según criterios predefinidos.

Palabras clave

Funciones ejecutivas, lectura, aprendizaje, desarrollo.

6 Para citar este artículo: Restrepo, G., Calvachi, L., Cano, I.C., \& Ruiz, A.L. (2019). Las funciones ejecutivas y la lectura: Revisión sistemática de la literatura. Informes Psicológicos, 19(2), pp. 81-94http://dx.doi.org/10.18566/ infpsic.v19n2a06 


\title{
Executive functions and reading: a systematic review of the literature
}

\begin{abstract}
Executive functions are high level cognitive processes, responsible for the planning, execution and evaluation that the subject makes of their own behavior. Their study is justified since they are involved in the processes of learning and child development and are the very nucleus of the social and adaptive behavior of the human being. Therefore, identifying the factors that intervene in their production has become an essential objective of the research that seeks to develop effective interventions, in the clinical and educational field, in children and adolescents. This article reviews the scientific literature that relates executive functions and academic performance with the reading learning process. The scientific articles on this topic, published from 2010 to 2015, are studied. Out of a total of 34 search results, 6 scientific publications were selected and analyzed according to predefined criteria.
\end{abstract}

Keywords

Executive functions, reading, learning, development.

\section{As funções executivas e a leitura: revisão sistemática da literatura}

\section{Resumo}

As funções executivas são processos cognitivos de alto nível responsáveis pelo planejamento, execução e avaliação que o sujeito faz em relação à conduta própria. Seu estudo é justificado já que se encontram envolvidas nos processos de aprendizagem e desenvolvimento infantil e constituem 0 núcleo do comportamento social e adaptativo do ser humano. Por conseguinte, identificar os fatores que intervém no desenvolvimento destas funções tem-se tornado em um objetivo essencial das pesquisas que procuram desenrolar intervenções eficazes no campo clínico e educativo, em crianças e adolescentes. Este artigo faz uma revisão da literatura cientifica que relaciona as funções executivas e 0 desempenho acadêmico com a aprendizagem da leitura. Foram revisados artigos científicos que tratam este tópico, publicados desde 2010 até 2015. De um total de 34 resultados de pesquisa, 6 publicações cientificas foram selecionadas e analisadas de acordo com critérios predefinidos.

Palavras chave Funções executivas, leitura, aprendizagem, desenvolvimento. 


\section{ntroducción}

Aprender a leer implica el empleo de una serie de habilidades cognitivas de alto nivel como la atención, la memoria, el lenguaje y la abstracción, entre otras, las cuales dependen de distintas estructuras cerebrales, cuyo estudio ha sido exhaustivo en los años recientes. Estas habilidades cognitivas, a menudo englobadas bajo el nombre de meta cognición, han llamado la atención de investigadores y académicos de forma reciente, y particularmente un conjunto de procesos cognitivos de alto nivel conocidos como funciones ejecutivas (Goldstein \& Naglieri, Princiotta, \& Otero, 2014; Meltzer, 2010). Existen diversas investigaciones que vinculan de forma directa a las funciones ejecutivas con el aprendizaje de la lectura y la literatura científica también registra algunos estudios que relativizan estas relaciones (Allan \& Lonigan, 2011; Fuhs \& Day, 2011).

Las funciones ejecutivas hacen parte de un conjunto de funciones mentales de alto nivel que nos permiten planificar nuestras tareas y alcanzar nuestros objetivos en situaciones y contextos no rutinarios (Olson \& Luciana, 2008). Éstas intervienen, por consiguiente, en los procesos de aprendizaje y en el desempeño escolar en todas las etapas de la vida (Luciana, Conklin, Hooper \& Yarger, 2005; Escobar, Zabala \& Rozo, 2008). Además de esto, dado su carácter complejo, las funciones ejecutivas se han implicado en la habilidad para alcanzar metas y objetivos específicos, que no se alcanzan con las estrategias rutinarias de solución de problemas, utilizadas en contextos corrientes (Barkley, 1997). De acuerdo a varios autores, las funciones ejecutivas se organizan jerárquicamente y empiezan a desarrollarse a partir del primer año de vida (Diamond, 1990), continuando su desarrollo durante el periodo preescolar (Carlson, 2005; Garon, Bryson \& Smith, 2008) hasta la adultez (Anderson, 2008).

La noción de funciones ejecutivas engloba procesos diversos como la inhibición de respuestas automáticas, la flexibilidad cognitiva y la planificación motora. Según Denckla (1996), la capacidad para inhibir una respuesta inapropiada emerge muy temprano en la vida y contribuye al desarrollo ulterior de procesos más complejos como el razonamiento, la planificación de las acciones y la resolución de problemas. En el adulto, las funciones ejecutivas dependen de una red de conexiones cerebrales que implican el córtex prefrontal, un sector del cerebro que madura lentamente durante la infancia, alcanza su plena maduración en la adolescencia y continúa desarrollándose a lo largo de la vida adulta (de Haan, 2013). Existe un amplio acuerdo entre los investigadores sobre la existencia de tres funciones ejecutivas de base, a saber, la inhibición, la flexibilidad y la actualización cognitiva, que tendrían como factor común la utilización permanente de la memoria de trabajo (Best \& Miller, 2010; Diamond, 2013; Miyake e al., 2000). La inhibición se refiere a la capacidad para ignorar estímulos irrelevantes para una tarea específica. Por su parte, la flexibilidad y la actualización cognitiva son las habilidades que permiten cambiar la atención de una actividad cognitiva a otra, conservando en la memoria de trabajo los 
aspectos esenciales de la tarea solicitada. Por ejemplo, en el medio escolar, los niños deben pasar de una tarea a otra dependiendo de los contenidos revisados (lenguaje, matemáticas o historia). En este contexto, la lectura exige un cambio constante de actividad cognitiva puesto que el niño debe enfocarse en una palabra o en una frase asociándola con otras palabras ya leídas y conservadas en la memoria de trabajo (Cartwright, 2015).

Lo explicado en los parágrafos precedentes sugiere que, como aprendizaje, la lectura es un proceso muy complejo. La persona que aprende a leer debe pasar por una serie de etapas que, gracias a la práctica, le permiten automatizar el reconocimiento de los grafemas que forman parte de las palabras, la decodificación de los símbolos para transformarlos en palabras, la decodificación de las palabras y el acceso al significado en la memoria a largo plazo (Mayer, 2010). Es así como en la lectura intervienen procesos de codificación, recodificación y comprensión. La codificación y recodificación permiten el acceso al léxico, es decir, el reconocimiento de palabras, pero para los procesos de comprensión lectora, el lector debe recurrir a los procesos de análisis sintáctico y semántico que permiten darle significado al texto que lee (Cartwright, 2012; 2015).

Es por ello que, en los últimos años, diferentes autores han insistido en el rol de las funciones ejecutivas en el desempeño lecto-escritor. Por ejemplo, Altemeier, Jones, Abbott y Berninger (2006) realizan un estudio en el que analizan la relación entre el funcionamiento ejecutivo y el desempeño lector, en alumnos de $3^{\circ}$ y $5^{\circ}$ Grado en escuelas públicas de EEUU. Los resultados muestran que las funciones ejecutivas contribuyen en el proceso de lectoescritura. En la misma dirección, McClelland et al. (2007) evalúan el funcionamiento ejecutivo y el desarrollo de la lectoescritura de niños de 5-6 años. Los resultados sugieren que el funcionamiento ejecutivo es un buen predictor del desempeño lector en la edad escolar. Desde una perspectiva predictiva igualmente, Dice y Schwanenflugel (2012) realizan una investigación, con niños/as de 4-6 años en escuelas públicas de Estados Unidos, cuyos resultados sugieren que el funcionamiento ejecutivo, y más específicamente el control inhibitorio, tiene un efecto sobre el aprendizaje posterior de la lectura.

En otro orden de ideas, las funciones ejecutivas, según la literatura científica, también se relacionan con la memorización, la meta cognición, el aprendizaje, el razonamiento, la resolución de problemas y la regulación del comportamiento (Tirapu-Ustárroz, \& Muñoz-Céspedes, 2005). Éstas empiezan a desarrollarse a temprana edad y se asocian al desarrollo del lenguaje oral, y más específicamente con el desarrollo del lenguaje egocéntrico que los niños interiorizan y utilizan para guiarse a sí mismos a través de cada tarea (Alarcón-Rubio, SánchezMedina, \& Prieto-García, 2014). La regulación del comportamiento por medio del lenguaje interior constituye entonces un proceso de gran importancia para el aprendizaje escolar. Un déficit importante en estos aspectos conllevaría a problemas de aprendizaje y de convivencia que afectan el clima interpersonal y deterioran el sistema de enseñanza-aprendizaje en el salón de clase. 
Por ejemplo, los comportamientos antisociales, el racismo, la violencia de género, el bullying o el acoso escolar son sin duda la expresión de las dificultades en el desarrollo y la maduración de las funciones ejecutivas, actividades cada día más frecuentes en los escenarios educativos y que ponen en evidencia la importancia de trabajar en el desarrollo de las funciones ejecutivas desde una perspectiva educativa y de prevención. La existencia de estas problemáticas en la escuela demuestra además que los procesos que se han adelantado a favor de la convivencia no han tenido el impacto esperado en el sector educativo (Estudio técnico de insuficiencia educativa del Departamento de Nariño, 2015).

El desarrollo del lenguaje, la conciencia fonológica y la lectura tienen entonces un impacto importante sobre el desarrollo de las funciones ejecutivas (Diamond, 2010). Sin embargo, estos vínculos y este impacto, puestos de manifiesto en las investigaciones reseñadas previamente, no ha sido explorado de manera directa por ninguno de los autores que se interesan en el tema, lo que justifica nuestra investigación, dado el enorme impacto social y científico que podría tener este conocimiento. En otras palabras, el desarrollo de estrategias eficaces, que permitan a los padres y profesores potencializar el desarrollo de las funciones ejecutivas en los niños, tendría un impacto muy importante en la disminución de problemáticas psicosociales que tienen dentro de sus factores predisponentes el desarrollo deficitario o atípico de las funciones mencionadas. El objetivo fundamental de esta investigación es hacer una revisión sistemática de la literatura sobre las relaciones entre las funciones ejecutivas y la lectura en los niños en edad escolar.

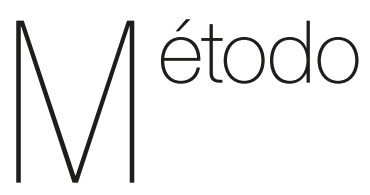

Las Revisiones Sistemáticas de la Literatura son un tipo de investigación observacional y retrospectiva que sintetizan los resultados de múltiples investigaciones primarias sobre un sujeto específico. Este tipo de investigación utiliza un método riguroso, explícito y reproductible, con criterios de inclusión y exclusión definidos con anterioridad, con el fin de permitir la identificación, la evaluación crítica y la síntesis de varios estudios previos, teniendo como objetivo primordial el responder a una pregunta específica de investigación. Es decir, que las revisiones sistemáticas son un tipo de investigación científica que tiene como propósito integrar de forma objetiva y sistemática los resultados de los estudios empíricos sobre un determinado problema de investigación, con el objetivo de establecer el 'estado del arte' en ese campo de estudio y proveer de esta manera una síntesis racional de la investigación sobre el tema estudiado (Martin, Renaud \& Dagenais, 2013).

\section{Criterios de exclusión e inclusión y estrategia general de la búsqueda}

Para realizar esta revisión sistemática de la literatura, se han definido varios 
criterios de exclusión e inclusión esenciales. Los artículos debían: (1) tratar de niños (por ejemplo, se excluyeron publicaciones sobre funciones ejecutivas y lectura en adultos); (2) Dado que esta investigación se enmarca en la perspectiva de estudiar el desarrollo infantil, los artículos retenidos debían tratar sobre el desarrollo de los procesos cognitivos de interés, principalmente las funciones ejecutivas y la lectura; (3) la búsqueda permitió identificar principalmente meta análisis, revisiones sistemáticas de literatura y ensayos controlados randomizados sobre los temas de interés; (4) finalmente, todas las publicaciones debían corresponder a artículos científicos. Por consiguiente, se excluyeron artículos publicados en revistas profesionales o sin comités de lectura.

\section{Procedimiento de búsqueda de datos}

Reflejando los criterios antes mencionados, los operadores booleanos (AND, OR, AND NOT) se combinaron con palabras clave para formar oraciones de búsqueda. Se ensayaron muchas combinaciones para descartar tantos artículos "no deseados" como fuese posible, pero también asegurándonos de que los artículos clave conocidos no se excluyeran de los resultados. Por lo tanto, la búsqueda fue intencionalmente más inclusiva que exclusiva.

La búsqueda en línea de las publicaciones científicas se llevó a cabo utilizando las plataformas MEDLINE, PsycINFO, ERIC y COCHRANE, que incluyeron todas las bases de datos electrónicas relevantes sobre neurociencia, psicología, educación y desarrollo infantil, con el fin de asegurar la inclusión de todas las revistas pertinentes.

\section{Selección de artículos}

Para seleccionar los artículos en todos los motores de búsqueda, se leyeron primero todos los resúmenes para excluir los artículos que no cumplían con los criterios establecidos, es decir, aquellos no excluidos con las frases de la búsqueda booleana. Los artículos restantes fueron leídos con una atención muy particular en las secciones de metodología, discusión y conclusiones. Esta lectura permitió, cuando se cumplieron todos los demás criterios de inclusión, comprender si las interpretaciones y conclusiones de los autores proporcionaron o no información sobre las características fundamentales de las funciones ejecutivas y la lectura, así como los vínculos entre estos dos procesos.

Por ejemplo, se excluyeron artículos cuando los resultados de la investigación condujeron a una discusión únicamente sobre las funciones ejecutivas y su relación con los trastornos del aprendizaje o del comportamiento. Este pre-análisis cualitativo de las secciones de discusión sirvió para construir una cuadrícula de selección final, que refleja la importancia ponderada de los artículos en las disciplinas siguientes: psicología, neurociencias y educación. Por último, para asegurar un acuerdo completo entre los investigadores, se estableció un procedimiento previo y sencillo de selección, permitiendo que los autores ajustaran los 
pre-ensayos y discutir sus diferentes opciones (ver Tabla 1).

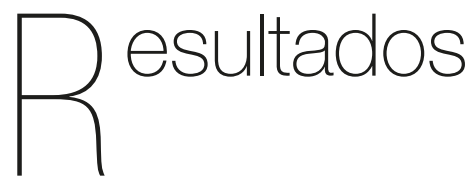

La frase de búsqueda booleana generó un total de 34 artículos en el motor de búsqueda de la biblioteca de la universidad. Del total de artículos, se seleccionaron seis, pues solo este número cumplió con los criterios de búsqueda. De los artículos encontrados, uno estaba escrito en español y 5 en inglés, los cuales se constituyeron en nuestro corpus, siguiendo el proceso de búsqueda y selección señalados.

Todos los artículos se dividieron inicialmente en dos grupos en relación con sus objetivos principales: (1) estudios de desarrollo; (2) estudios centrados en factores situacionales, sociales y emocionales. Se identificó un estudio de desarrollo cuando se compararon los resultados de aprendizaje basados en retroalimentación entre grupos con diferentes edades o cuando se anunció claramente un enfoque de desarrollo.

La Tabla 1 resume las características de los artículos que presentan un estudio de desarrollo $(n=6)$. Estos artículos fueron publicados desde 2005 a 2015.

Tabla 1.

Características de los artículos revisados

\begin{tabular}{|c|c|c|c|c|c|}
\hline Autores & Año & Objetivo principal de la investigación & $\begin{array}{l}\text { Participantes } \\
\text { (Rango de edad } \\
\text { y media) }\end{array}$ & Método & Resultados \\
\hline $\begin{array}{l}\text { Villalón } \\
\text { et al. }\end{array}$ & 2011 & $\begin{array}{l}\text { Evaluar los resultados de una intervención focalizada } \\
\text { en el aprendizaje temprano de lectura y escritura de } \\
\text { niños con riesgo social. Especialmente en el programa } \\
\text { se examinarán los resultados de un programa de de- } \\
\text { sarrollo profesional que incluye capacitación y acom- } \\
\text { pañamiento experto a los profesores en las áreas de } \\
\text { conocimiento del alfabeto, reconocimiento visual de } \\
\text { palabras, escritura emergente y comprensión oral en } \\
\text { los niños. }\end{array}$ & $\begin{array}{c}\text { Total de } \\
\text { Niños: } 339 \\
195 \text { Mujeres } \\
144 \text { Hombres } \\
\text { M }=5.29\end{array}$ & $\begin{array}{l}\text { Diseño cua- } \\
\text { siexperimental }\end{array}$ & $\begin{array}{l}\text { Existen diferencia significativa entre los } \\
\text { niños del grupo de comparación en tres } \\
\text { de los aspectos evaluados, a saber: cono- } \\
\text { cimiento del alfabeto, escritura emergen- } \\
\text { te y reconocimiento visual de palabras. } \\
\text { Sin embargo. No se observó diferencia } \\
\text { entre ambos grupos en los avances de } \\
\text { comprensión oral. }\end{array}$ \\
\hline
\end{tabular}

Continúa 
Continuación

\begin{tabular}{|c|c|c|c|c|c|}
\hline Autores & Año & Objetivo principal de la investigación & $\begin{array}{l}\text { Participantes } \\
\text { (Rango de edad } \\
\text { y media) }\end{array}$ & Método & Resultados \\
\hline $\begin{array}{l}\text { Jacob \& } \\
\text { Parkinson }\end{array}$ & 2015 & $\begin{array}{l}\text { Revisar sistemáticamente la asociación entre la fun- } \\
\text { ción ejecutiva y el rendimiento estudiantil tanto en } \\
\text { lectura como en matemáticas y evaluar críticamente } \\
\text { la asociación casual entre las dos, utilizando técnicas } \\
\text { meta analíticas. }\end{array}$ & $\begin{array}{l}\text { Edades de } \\
2 \text { a } 18 \text { años } \\
\quad M=8\end{array}$ & Metaanalisis & $\begin{array}{l}\text { Existe una asociación moderna entre la } \\
\text { función ejecutiva y el logro, que no distin- } \\
\text { gue por componente de la función ejecuti- } \\
\text { va, edad o tipo de medición, no encuentra } \\
\text { evidencia contundente de que exista rela- } \\
\text { ción causal entre las dos. }\end{array}$ \\
\hline $\begin{array}{l}\text { Booth, } \\
\text { Boyle, } \\
\text { \& Kelly }\end{array}$ & 2010 & $\begin{array}{l}\text { Diferentes estudios han evidenciado que los niños con } \\
\text { dificultades de lectura presentan déficit en la función } \\
\text { ejecutiva y otros estudios no han detectado tal déficit. } \\
\text { La finalidad del presente meta-análisis es determinar } \\
\text { si estos hallazgos discrepantes pueden ser explicados } \\
\text { por las diferencias en las tareas de la función ejecutiva } \\
\text { que se utilizan en los estudios. }\end{array}$ & $\begin{array}{l}\text { La mediana de un } \\
\text { número de partici- } \\
\text { pantes por estudio } \\
\text { fue de } 52 \text { (rango } \\
20-665 \text { ). Edad } 75 \\
\text { y } 179 \text { meses M } \\
=126 \text { meses }\end{array}$ & Metaanalisis & $\begin{array}{l}\text { Los niños con dificultades de lectura } \\
\text { tienen impedimentos al respecto de las } \\
\text { funciones ejecutivas. Las discrepancias } \\
\text { en la literatura podrían ser un producto de } \\
\text { la gran variedad de tareas de evaluación } \\
\text { que se están utilizando y también de que } \\
\text { las diferencias entre los criterios utilizados } \\
\text { para seleccionar dificultades de lectura } \\
\text { pueden dar lugar a nuevas variaciones. }\end{array}$ \\
\hline $\begin{array}{l}\text { Houdé, } \\
\text { Rossi, } \\
\text { Lubin, \& } \\
\text { Joliot }\end{array}$ & 2010 & $\begin{array}{l}\text { Analizar los datos de resonancia magnética funcional } \\
\text { IRMF obtenidos en la década 1999-2008 en más de } \\
800 \text { niños y adolescentes, en tres sistemas básicos } \\
\text { de desarrollo cognitivo y aprendizaje escolar: habilida- } \\
\text { des numéricas, lectura y funciones ejecutivas (Control } \\
\text { cognitivo). }\end{array}$ & $\begin{array}{l}842 \text { participantes. } \\
\text { Edades 4-17 años }\end{array}$ & Metaanalisis & $\begin{array}{l}\text { Los análisis muestran que los niños se in- } \\
\text { volucran principalmente en regiones fron- } \\
\text { tales al resolver tareas numéricas. Con la } \\
\text { edad, podría pasar (durante estas tareas), } \\
\text { de depender de la corteza parietal. }\end{array}$ \\
\hline $\begin{array}{l}\text { Yeniad, } \\
\text { Malda, } \\
\text { Mesman, } \\
\text { Van ljzen- } \\
\text { doorn, \& } \\
\text { Pieper }\end{array}$ & 2013 & $\begin{array}{l}\text { Investigar si la flexibilidad cognitiva está significativa- } \\
\text { mente relacionada con el rendimiento en matemáticas } \\
\text { y lectura en los niños. }\end{array}$ & $\begin{array}{l}\text { Total de niños: } \\
\quad 2330\end{array}$ & Metaanálisis & $\begin{array}{l}\text { Los niños con mayor flexibilidad cognitiva, } \\
\text { muestran mejor rendimiento en mate- } \\
\text { máticas y lectura. La literatura muestra } \\
\text { asociación significa y sustancial entre la } \\
\text { flexibilidad cognitiva y la inteligencia. El } \\
\text { cambio en la capacidad y la flexibilidad } \\
\text { entre reglas diferentes, estrategias y } \\
\text { tareas, es un indicador directo del logro } \\
\text { académico, sin tener en cuenta las varia- } \\
\text { ciones en las muestras y los diferentes } \\
\text { procedimientos. }\end{array}$ \\
\hline $\begin{array}{l}\text { Kudo, } \\
\text { Lussier, } \\
\text { Swanson }\end{array}$ & 2015 & $\begin{array}{l}\text { Sintetizar las diferencias cognitivas entre niños con y } \\
\text { sin discapacidad de lectura. }\end{array}$ & $\begin{array}{l}\text { Niños entre } \\
\text { 72-191 meses }\end{array}$ & Metaanalisis & $\begin{array}{l}\text { Abundante evidencia muestra que los } \\
\text { niños con discapacidad de lectura tie- } \\
\text { nen problemas en el procesamiento de } \\
\text { información fonológica. Algunos estudios } \\
\text { sugieren que la consciencia fonológica, } \\
\text { pueden estar involucrada en la adquisi- } \\
\text { ción de la lectura. }\end{array}$ \\
\hline
\end{tabular}

Fuente: elaboración propia

Resultados

\section{Estudios de desarrollo}

En el estudio de Villalón et al. (2011) titulado "Resultados de la enseñanza de estrategias de lectura y escritura en la alfabetización temprana de niños con riesgo social" se encuentra que existen diferencias significativas entre los niños del grupo experimental y el grupo control, en tres de los aspectos evaluados, a saber: conocimiento del alfabeto, escritura emergente y reconocimiento visual de palabras. Sin embargo, no se observaron diferencias entre ambos grupos en lo que respecta a la comprensión oral. Teniendo en cuenta la importancia de la alfabetización temprana sobre el aprendizaje de la lectura, se concluye que en los 
colegios no existen las condiciones para hacer este tipo de intervenciones, pues los docentes carecen del conocimiento que se requiere para hacerlo. Igualmente, se sugiere que existe una asociación moderada entre las funciones ejecutivas y el aprendizaje de la lectura, sin que pueda distinguirse el tipo específico de función ejecutiva, la edad o tipo de medición de esta función. Por consiguiente, no se encuentra evidencia contundente de que exista relación causal entre estos dos procesos. En lo concerniente a las restantes publicaciones, la mayoría de estudios evaluaron la función ejecutiva y los logros tanto en matemáticas como en lectura, sin embargo, se aprecia una mayor asociación entre funciones ejecutivas y matemática que en lectura particularmente en el estudio de Jacob y Parkinson (2015). En otro orden de ideas, el metaanálisis de Booth, Boyle y Kelly (2010) muestra que los niños con dificultades de lectura tienen bajo desempeño en tareas relacionadas con las funciones ejecutivas. Sin embargo, el tamaño del efecto varió considerablemente en todos los 180 resultados analizados (.57, SE.03), sugiriendo que el compromiso de las funciones ejecutivas no es uniforme, que el tipo de tarea empleada y las demandas subyacentes a la tarea, así como las puntuaciones del coeficiente intelectual $\mathrm{Cl}$, pueden influir en los resultados observados. Por el contrario, la edad y el género de los participantes y el tipo de lectura no parece tener una influencia significativa sobre los resultados. Los hallazgos de este metaanálisis indican que los niños con dificultades de lectura tienen problemas en el desarrollo de las funciones ejecutivas pero que las discrepancias encontradas pueden también deberse al tipo de literatura analizada. Por ejemplo, los niños con mayor flexibilidad cognitiva muestran un mejor rendimiento en matemáticas y lectura, lo que lleva a pensar que el desarrollo de la competencia lectora y de las habilidades matemáticas dependen mucho de una capacidad cognoscitiva mucho más general. En ese sentido, la literatura científica muestra, en general, una asociación significativa y sustancial entre la flexibilidad cognitiva y la inteligencia. Esto quiere decir que la capacidad de adaptación cognitiva y la flexibilidad para identificar los cambios en las reglas, las estrategias y las tareas, suele ser un indicador directo del logro académico (Yeniad et al., 2013).

Numerosos estudios muestran igualmente que los niños con dificultades de lectura tienen problemas en el análisis y procesamiento de la información fonológica. Algunos de estos estudios sugieren incluso que la consciencia fonológica puede estar involucrada en la adquisición de la lectura. Por ejemplo, en el estudio de Kudo, Lussier y Swanson (2015) las pruebas que evalúan la consciencia fonológica (construcción de palabras, lectura de pseudo palabras y reconocimiento ortográfico) mostraron claramente las debilidades, en esta función, de los niños que presentan un trastorno del aprendizaje de la lectura. Por el contrario, estos autores no encontraron diferencias significativas en cuanto al género y la procedencia étnica. Ellos argumentan que las regiones frontales se activan al resolver tareas numéricas y de conciencia fonológica, lo que se explicaría por la importancia de estas regiones en el desarrollo de las funciones ejecutivas. Sin embargo, con la edad, podría darse un cambio en esta activación y el control de estas funciones podría pasar de la corteza frontal a la corteza parietal 
izquierda, un fenómeno que ha sido corroborado en estudios que analizan la activación cerebral en la realización de tareas numéricas y en pruebas que involucran la consciencia fonológica (Houdè, Rossi, Lubin \& Joliot, 2010).

Desde una perspectiva comparativa, Kudo et al. (2015) realizaron un meta-análisis en el cual sintetizaron las diferencias en el proceso cognitivo entre niños con y sin problemas de lectura. El estudio se centró en dos asuntos fundamentales: el primero en identificar los dominios que contribuyeron a la mayor diferencia entre niños con y sin problemas de lectura. El análisis factorial indicó que varios procesos cognitivos (memoria de trabajo verbal, memoria visual-espacial, funciones ejecutivas, velocidad de procesamiento, memoria a corto plazo y habilidades motrices perceptivas), así como los resultados de pruebas de conciencia fonológica (lectura de pseudopalabras, vocabulario, ortografía) contribuyeron con una variación significativa en los resultados globales. Es decir, que las dificultades observadas en estos niños no siempre se relacionaban con las funciones ejecutivas sino con otros procesos cognitivos diferentes. El segundo asunto analizado en la investigación de Kudo et al. (2015) tuvo que ver con la pregunta de si las diferencias de rendimiento entre los niños con y sin trastornos de lectura eran diferencias moderadas por el coeficiente intelectual, la edad, la etnia o el género. En cuanto a la etnia y el género, estos autores encontraron que pocos estudios de los elegidos informaron sobre los efectos específicos de esas variables $\mathrm{y}$, por tanto, su análisis no fue concluyente en modo alguno. Lo que sí se halló es que la edad cronológica no fue un moderador significativo del tamaño del efecto entre niños con y sin trastornos de lectura. Esto sugiere que, a pesar de la maduración en funciones ejecutivas, esperada con la edad, el efecto se mantiene indicando una relación importante entre las dificultades de lectura y los problemas en el desarrollo de las funciones ejecutivas. Por el contrario, el coeficiente intelectual sí moderó los resultados generales, dando más fuerza al argumento de que las funciones ejecutivas son un componente importante de la inteligencia y de la capacidad cognitiva general del individuo.

Sin embargo, estos resultados son contrarios a los encontrados por Booth et al. (2010), quienes en su metaanálisis arguyen que las funciones ejecutivas están siempre involucradas de forma constante en las dificultades de lectura, aunque el tamaño del efecto promedio (.57), varía considerablemente en todos los resultados. Igualmente, Booth et al. (2010) encontraron que la edad y el género de los participantes y la naturaleza del trastorno de lectura no tuvieron influencia en los tamaños del efecto.

Un metaanálisis más reciente aborda el asunto de una forma un poco diferente. Es así como Jacob y Parkinson (2015) se proponen revisar la asociación y relación causal entre funciones ejecutivas y rendimiento académico en lectura y matemáticas. Estos autores encontraron una asociación moderada entre la función ejecutiva y el logro académico que no difiere con respecto al tipo de función ejecutiva o a la edad. Efectivamente, ellos encuentran un tamaño de efecto de .30 entre las funciones ejecutivas y los resultados académicas, el cual no cambia con la edad o con los diferentes subcomponentes de la función ejecutiva 
(inhibición, control de atención, cambio de atención y memoria de trabajo), incluso haciendo mediciones en diferentes contextos (mediciones naturalistas versus mediciones hechas en el laboratorio). De forma interesante, a pesar de las hipótesis esgrimidas por el equipo, ellos encontraron sólo pruebas limitadas de que la asociación entre la función ejecutiva y los logros en las áreas evaluadas era más fuerte en matemáticas que en lectura. Pero cuando se examinó una medida global de la función ejecutiva (en lugar de los subcomponentes) no encontraron ninguna diferencia en la asociación entre función ejecutiva, lectura o matemáticas. Y al explorar las asociaciones por separado con los subcomponentes, se encontró mayor fuerza en la asociación entre la función ejecutiva y matemáticas en relación con el control atencional y la inhibición, pero no con la memoria de trabajo o la flexibilidad atencional. En conclusión, este estudio no muestra evidencia convincente de que exista una asociación causal entre las funciones ejecutivas y el desempeño en lectura o en matemáticas.

Por su parte, en otro metaanálisis, Yeniad et al. (2013) encontraron una asociación significativa entre la flexibilidad cognitiva de un lado y las habilidades en lectura y matemáticas, del otro lado. Estos autores consideran incluso que existe una relación significativa y sustancial entre funciones ejecutivas y rendimiento académico. Sin embargo, hubo cierta variación en las medidas de asociación entre la flexibilidad y el logro académico, lo cual no se relacionó con diferencias en la presentación de la tarea, o en los resultados académicos, en la edad de los niños, en el nivel de estudios o en el género. Ellos concluyen que la evidencia empírica que existe entre el funcionamiento ejecutivo y el rendimiento académico es ambigua. Los resultados de este estudio sugieren que la inteligencia mostraba asociaciones más fuertes con matemáticas que con la lectura. Por lo tanto, argumentan que la asociación entre flexibilidad cognitiva, habilidades académicas e inteligencia son de orden general y no se puede concluir una relación de causalidad. De esta forma, el resultado principal del meta-análisis indica que los niños con una mayor flexibilidad a nivel de representación conceptual (objetivos, reglas o estrategias para solución del problema) muestra un mejor rendimiento en matemáticas y la lectura.

Es importante contrastar estos resultados con lo encontrado por Villalón et al. (2011), quienes proponen intervenir precozmente, a través de la alfabetización temprana, sobre los procesos del aprendizaje de la lectura y la escritura, en niños expuestos a riesgo social. En este orden de ideas, Kudo et al. (2015) mostraron en su meta-análisis que los estudiantes con problemas de lectura tienen numerosos rezagos cognitivos en varias áreas en comparación con los estudiantes de lectura promedio, e identifican específicamente las áreas que necesitan intervención. Aquellas áreas específicamente encontradas que contribuyeron a entender las diferencias entre los niños con problemas de lectura y los lectores promedio estuvieron en habilidades de procesamiento fonológico, incluyendo el reconocimiento de palabras, así como áreas cognitivas y académicas incluyendo ortografía y vocabulario, memoria de trabajo verbal, memoria visual-espacial, procesamiento ejecutivo, y memoria a corto plazo. 


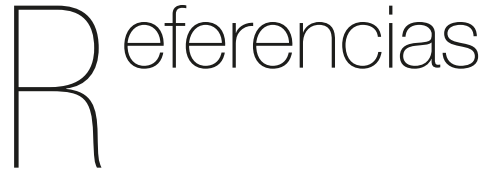

Alarcón-Rubio, D., Sánchez-Medina, J. A., \& Prieto-García, J. R. (2014). Executive function and verbal self-regulation in childhood: Developmental linkages between partially internalized private speech and cognitive flexibility. Early Childhood Research Quarterly, 29(2), 95-105.

Allan, N. P., \& Lonigan, C. J. (2011). Examining the dimensionality of effortful control in preschool children and its relation to academic and socioemotional indicators. Developmental Psychology, 47(4), 905-915.

Altemeier, L., Jones, J., Abbott, R. D., \& Berninger, V. (2006). Executive functions in becoming writing readers and reading writers: Note-taking and report writing in third and fifth graders. Developmental Neuropsychology, 29(1), 161-173.

Anderson, V., Anderson, P. J., Jacobs, R., \& Smith, M. S. (2008). Development and assessment of executive function: From preschool to adolescence. In $\mathrm{V}$. Anderson, R. Jacobs, \& P. J. Anderson (Eds.), Neuropsychology, neurology, and cognition. Executive functions and the frontal lobes: A lifespan perspective (pp. 123-154). Philadelphia, PA, US: Taylor \& Francis.

Barkley, R. A. (1997). Behavioral inhibition, sustained attention, and executive functions: Constructing a unifying theory of ADHD. Psychological Bulletin, 121(1), 65-94.
Best, J. R., \& Miller, P. H. (2010). A Developmental Perspective on Executive Function. Child Development, 81, 1641-1660.

Booth, I. N., Boyle, J. M. E. \& Kelly, S. W. (2010). Do task make a difference? Accounting for heterogeneity of performance of children with reading difficulties on tasks of executive function: Findings from a metaanalysis. British Journal of Developmental Psychology, 28, 133-176

Cartwright, K. B. (2012). Insights from Cognitive Neuroscience: The Importance of Executive Function for Early Reading Development and Education. Early Education \& Development, 23(1), 24-36

Cartwright, K. B. (2015). Executive skills and reading comprehension: A guide for educators. New York, NY, US: Guilford Press.

Carlson, S.M., Davis, A., \& Leach J.G. (2005). Less is More: Executive function and symbolic representation in preschool children. Psychological Science, 6, 609-616.

Conklin, H. M., Luciana, M., Hooper, C. J., \& Yarger, R. S. (2007). Working memory performance in typically developing children and adolescents: Behavioral evidence of protracted frontal lobe development. Developmental Neuropsychology, 31(1), 103-128.

De Haan, M. (2014). Attention and executive control. In D. Mareschal, B. Butterworth, \& A. Tolmie (Eds.), Educational Neuroscience (pp. 325-349). Hoboken, NJ: John Wiley \& Sons.

Denckla, M. B. (1996). A theory and model of executive function: A neuropsychological 
perspective. In G. R. Lyon \& N. A. Krasnegor (Eds.), Attention, memory, and executive function (pp. 263-278). Baltimore, MD, US: Paul H Brookes Publishing.

Diamond, A. (1990). Developmental time course in human infants and infant monkeys, and the neural bases of inhibitory control in reaching. Annals of the New York Academy of Sciences, 608, 637-676.

Diamond, A. (2010). The evidence base for improving school outcomes by addressing the whole child and by addressing skills and attitudes, not just content. Early Education and Development, 21(5), 780-793.

Diamond, A. (2013). Executive Functions. Annual Review of Psychology, 64, 135-168.

Dice, J. L., \& Schwanenflugel, P. (2012). A structural model of the effects of preschool attention on kindergarten literacy. Reading and Writing, 25(9), 2205-2222.

Escobar, M. R., Zabala, M. E. Z., \& Rozo, P. J. P. (2008). Perfil neuropsicológico de escolares con trastornos específicos del aprendizaje de instituciones educativas de Barranquilla, Colombia. Acta Neurológica Colombiana, 24(2), 63-73.

Funs, M. W., \& Day, J. D. (2011). Verbal ability and executive functioning development in preschoolers at head start. Developmental Psychology, 47(2), 404-416.

Garon, N., Bryson, S. E., \& Smith, I. M. (2008). Executive function in preschoolers: A review using an integrative framework. Psychological Bulletin, 134(1), 31-60.

Goldstein S., Naglieri J. A., Princiotta D., \& Otero, T. M. (2014). Introduction: a history of executive functioning as a theoretical and clinical construct. In S. Goldstein \& J.A. Naglieri (eds.), Handbook of Executive Functioning (pp. 3-12). New York, NY: Springer.

Houdé, O., Rossi, S., Lubin, A., \& Joliot, M. (2010). Mapping numerical processing, reading, and executive functions in the developing brain: An FMRI meta-analysis of 52 studies including 842 children. Develomental Science, 13(6), 876-885.

Jacob, R., \& Parkinson, J. (2015). The potential for school-based interventions that target executive function to improve academic achievement: A review. Review of Educational Research, 85(4), 512-552.

Kudo M. F., Lussier C. M., \& Swanson H. L. (2015). Reading disabilities in children: A selective meta-analysis of the cognitive literature. Research in Developmental Disabilities, 40, 51-62.

Luciana, M., Conklin, H.M., Hooper, C.J., \& Yarger, R.S. (2005). The development of nonverbal working memory and executive control processes in adolescents. Child Development, 76(3), 697-712.

Meltzer, L. (2010). Promoting executive function in the classroom. New York and London: Guilford Press

Martin, V., Renaud, J., \& Dagenais, P. (2013). Les normes de production des revues systématiques: Guide méthodologique. Francia: Institut national d'excellence en santé et en services sociaux (INESS).

McClelland, M., Cameron, C., Connor, C., McDonald, F., Carrie, L., Jewkes, A. \& Morrison, F. (2007). Links between behavioral regulation and preschoolers' literacy, vocabulary, and math skills. 
Developmental Psychology, 43(4), Tirapu-Ustárroz, J. \& Muñoz-Céspedes, J.

947-959.

Mayer, R.E. 2010. Applying the science of learning to medical education. Medical Education, 44, 543-549.

Miyake, A., Friedman, N., Emerson, M., Witzki, A., Howerter, A., \& Wager, T. D. (2000). The unity and diversity of executive functions and their contributions to complex "frontal lobe" tasks: A latent variable analysis. Cognitive Psychology, 41, 49-100.

Olson, E., \& Luciana, M. M. (2008). The development of prefrontal cortex functions in adolescence: theoretical models and a possible dissociation of dorsal versus ventral subregions. In C. A. Nelson, \& M. M. Luciana (Eds.), The Handbook of Developmental Cognitive Neuroscience, (2nd ed.). MIT Press.
(2005). Memoria y funciones ejecutivas. Revista de Neurología, 41(8), 475-84.

Villalón, M., Förster, C., Cox, P., RojasBarahona, C., Valencia, E., \& Volante, P. (2011). Resultados de la enseñanza de estrategias de lectura y escritura en la alfabetización temprana de niños con riesgo social. Estudios sobre Educación, 21, 159-179.

Yeniad, N., Malda, M., Mesman, J., van IJzendoorn, M. H., \& Pieper, S. (2013). Shifting ability predicts math and reading performance in children: A meta-analytical study. Learning and Individual Differences, 23, 1-9. 